\title{
Money And The Scale of CoOperation*
}

\author{
Maria Bigoni \\ University of Bologna
}

\author{
Gabriele Camera \\ Chapman University \\ \& University of Basel
}

December 19, 2015

\author{
Marco Casari \\ University of Bologna \\ \& IZA
}

\begin{abstract}
This study reveals the existence of a causal link between the availability of money and an expanded scale of interaction. We constructed an experiment where participants chose the group size, either a low-value partnership or a high-value group of strangers, and then faced an intertemporal cooperative task. Theoretically, a monetary system was inessential to achieve cooperation. Empirically, without a working monetary system, participants were reluctant to expand the scale of interaction; and when they did, they ended up destroying surplus compared to partnerships, because cooperation collapsed in large groups. This economic failure was reversed only when participants managed to concurrently develop a stable monetary system.
\end{abstract}

Keywords:

Endogenous institutions, experiments, repeated games, strategic uncertainty.

\footnotetext{
* We thank Vincent Bourke and Megan Luetje for outstanding research support, and seminar participants at the University of Bologna, Goethe University in Frankfurt, Tilburg University, GATE (Lyon), and at the Workshop in Behavioral Public Economics in Vienna. G. Camera acknowledges partial research support through the NSF grant CCF-1101627. M. Casari acknowledges financial support through the ERC Starting Grant number 241196.

Correspondence address: Gabriele Camera, Economic Science Institute, Chapman University, One University Dr., Orange, CA 92866; e-mail: camera@chapman.edu.
} 


\section{Introduction}

Large scale cooperation is central to economic development but presents formidable challenges (Kimbrough et al., 2008; North, 1991). How can agents succeed in widening the scale of economic cooperation? Suitable institutions are of primary importance in overcoming these challenges. The literature has focused on monitoring, enforcement, communication, and punishment (Greif, 2006; Milgrom et al., 1990). The present study is about money. We consider a situation where agents can choose between a stable partnership or higher-value interactions in large groups of strangers. In a laboratory setting, we show that a monetary system is behaviorally crucial in widening the scale of cooperation, even when it is theoretically inessential.

History provides anecdotal evidence of a relation between the scale of economic cooperation and the availability of reliable monetary systems. For example, thirteenth century trade in Europe flourished at the time Genoa and Florence returned to strike gold coins (Lopez, 1971), and eighteenth century commerce in the West relied on the Spanish dollar. These observations do not constitute evidence of a causal link between emergence of monetary institutions and large scale cooperation. The expansion of trade may stem from superior legal institutions or military might, and not from monetary systems; conversely, the failure to expand the scale of cooperation may be due to low returns from trade or technological factors, and not due to absence of monetary systems. The advantage of the experimental methodology is that we can suppress institutional and environmental confounding factors that characterize field data, and understand what principles are in operation (Plott, 2001).

Consider a setup that captures the principle behind the typical monetary trade: a producer gives a valuable good to a consumer in exchange for a to- 
ken, which is a symbolic object. The good is produced at a cost below its consumption value and though the token has no intrinsic value, it is storable for exchange in future rounds. In this situation, there are gains from trade. We construct a repeated helping game where agents face a sequence of pairwise encounters in which their role alternates between producer and consumer (Townsend, 1980). In each encounter the producer suffers a small cost to "help" the consumer by giving her the good. The consumer can concurrently transfer a token to the producer. Helping maximizes benefits in the pair, irrespectively of whether a token is transferred or not. This theoretical setup is contrasted to a situation where tokens are not present and therefore the consumer has nothing to transfer to the producer (Ellison, 1994; Friedman, 1971; Kandori, 1992). In all setups, cooperation amounts to an intertemporal exchange of help.

For each setup, we study the sustainability of cooperation both theoretically and in a controlled experiment. The experiment has two conditions, one in which a monetary system can spontaneously emerge, and another in which it cannot (Bigoni et al., 2014). In each condition, the scale of cooperation is endogenous. Participants can choose to interact either with a fixed counterpart in a partnership (Dal Bó and Fréchette, 2011; Palfrey, 1994), or in a large group of strangers (Camera et al., 2013). This choice is meaningful because by design help carries a greater benefit in large groups than in partnerships.

Having repeated opportunities to help gives rise to a social dilemma that is especially conspicuous in large groups, where interaction is impersonal and reciprocity impossible (Fehr and Gächter, 2000; Gächter and Hermann, 2011). Payoffs are maximized under full cooperation, i.e. when every producer helps in every round. However, participants may refuse to help to avoid an immediate cost, and this inevitably minimizes payoffs for everyone in the group. 
Folk theorem-type of results imply the existence of multiple equilibria (Ellison, 1994; Kandori, 1992). This situation is characterized by strategic uncertainty (Van Huyk et al., 2007), which makes it harder to attain efficient outcomes in large groups (Weber, 2006). If participants succumb to short-run opportunistic temptations, then there will be little or no cooperation. However, full cooperation can also spontaneously emerge if participants succeed in coordinating actions intertemporally through a norm of mutual help. This requires sufficient trust that help given today will be reciprocated by strangers in future encounters. When tokens are available, cooperation can also be achieved by developing a convention of monetary trade. This requires trusting that strangers will help in exchange for a token in future encounters (Camera and Casari, 2014). However, by construction monetary trade is neither necessary nor sufficient to achieve cooperation. Contrasting the two conditions thus reveals whether or not the availability of symbolic media of exchange behaviorally influences the chosen scale of interaction, and of cooperation.

There is a growing experimental literature on money as a means of payment, starting with the early contributions of McCabe (1989), and of Lian and Plott (1998). In particular, this paper is related to our previous experiments about decentralized, fiat monetary systems: Camera and Casari (2014) studies the coordination role of monetary exchange, while Camera et al. (2013) studies cooperation with and without money in small and large groups, offering an evolutionary explanation for the use of money. Duffy and Puzzello (2014) also exogenously manipulates group size in reference to a specific monetary model. The endogenous choice of the scale of interaction is an original aspect of the present study, as previous experiments study group formation only without money (Ahn et al., 2009). 


\section{The game}

The experiment has a Control and a Tokens condition. In the Control condition, participants play a "helping game" in pairs composed of a producer and a consumer. Each producer starts with $d=6$ consumption units (CUs) and can choose to help ("give help") or not ("no help"). The consumer has $d-l=3$ CUs. Helping yields a payoff of 0 CUs to the producer and a payoff of $k>2 d-l$ CUs to the consumer; the net benefit from help is $k-2 d+l$ CUs.

Participants play this game repeatedly, in "cycles" that last nineteen rounds on average. In each round, half of the participants are consumers and half producers. Roles are randomly assigned in the first round, and deterministically alternate in the following rounds. Participants know they play sixteen rounds and from round sixteen on they play an additional round with 75\% probability, otherwise the cycle ends (Fréchette and Yuksel, 2013; Palfrey, 1994; Roth and Murnighan, 1978). CUs cumulate across rounds, and are converted into dollars at the end of the session. This set-up captures the essence of an interaction, in which there are gains from intertemporal trade.

A session includes six cycles. In each cycle, participants interact either in partnerships or large groups of 12 or 24 individuals. In a partnership, the counterpart is fixed throughout a cycle. In large groups, the counterpart is randomly chosen in every round, and identities remain undisclosed; hence, individuals interact as strangers. There is public monitoring of defections: strangers can observe whether or not every producer in their group helps.

Benefits are greater in large groups $(k=18)$ than in partnerships $(k=15)$. If no one cooperates, then average per-capita payoffs are $4.5 \mathrm{CUs}$ both in partnerships and large groups. Instead, under full cooperation they reach 7.5 CUs in partnerships, and 9 CUs in large groups. The difference between per- 
capita payoffs attained and the 4.5 CUs baseline measures how much surplus is created in a round of play. Hence, expanding the scale of interaction can be beneficial since large groups can create $50 \%$ more surplus than partnerships (4.5 CUs vs. 3 CUs). However, surplus creation is not an automatic processit depends on the degree of cooperativeness actually achieved in the group. We assess a group's success in creating surplus by measuring economic efficiency, defined as the proportion of surplus created by the group in the average round of play relative to the maximum potential of $4.5 \mathrm{CUs}$. Efficiency is directly proportional to the cooperation rate in the group. Efficiency is zero when no one cooperates - in groups of any size - while if everyone cooperates it reaches $67 \%$ (=3 out of $4.5 \mathrm{CUs}$ ) in partnerships and $100 \%$ (=4.5 out of $4.5 \mathrm{CUs}$ ) in large groups. Rational, self-interested participants can attain full cooperation by following a simple rule of conduct: they help as long as everyone else does the same; otherwise, they will never help again (Ellison, 1994; Friedman, 1971; Kandori, 1992).

In the ToKens condition, we add symbolic and intrinsically worthless objects - or "tokens" - which cannot be redeemed for CUs or dollars, and have no reference to outside currencies. This expands the strategy space, by introducing the possibility of trading help through a direct mechanism (see Table A1 in Appendix A). The supply of tokens is fixed: in round one, every consumer has one token and producers have none. This introduces the possibility of fiat monetary exchange. The consumer has three alternative actions: carry over the token to the next round ("Do nothing"); unilaterally "transfer a token"; or "buy help" in exchange for a token. The producer can "give help" or not - as in the ConTROL condition-but can also "sell help" in exchange for a token. Choices are made simultaneously and without communication.

The two possible payoff configuration are the same as in the CONTROL 
condition. The payoffs are $0 \mathrm{CUs}$ for the producer, and $k$ CUs for the consumer, when the producer helps unconditionally or help is exchanged for a token. Otherwise the payoffs are 6 CUs for the producer, and 3 CUs for the consumer. At the end of each round, a participant observes the outcome in the pair but not the action of the opponent. If a consumer has no tokens, he has no actions to take, and the producer can only choose whether or not helping unconditionally: hence the decision situation is identical to the CONTROL condition. Token holdings are partially observable by the opponent: in every pair, each player can verify whether the opponent has either 0 or at least one token; the exact number is unobservable in order to preserve anonymity and to reduce the cognitive load.

In the ToKens condition the cooperative equilibrium can also be sustained through a monetary trade convention, where all consumers buy help, and all producers sell help. However, trading tokens for help is theoretically unnecessary to sustain full cooperation. The Tokens condition neither precludes the adoption of the social norm of cooperation, nor forces participants to use tokens; it simply expands the strategy set, without removing any equilibria of the CONTROL condition or adding more efficient equilibria.

Each session consists of a Training Phase (cycles 1-4) and a Selection Phase (cycles 5-6). Training Phase interaction exogenously alternates across cycles between partnerships and groups of 12. Instead, the scale of interaction in the Selection Phase is endogenous. Session participants express a preference between partnerships and groups of 12 before cycles 2-5 start, and between partnerships and a group of 24 before cycle 6 starts. The majority of preferences determines the scale of interaction in cycles 5 and 6 , respectively (see Section 4 for details). 


\section{Theoretical considerations}

In this Section, we show the existence of a fully cooperative equilibrium under both experimental conditions. These considerations apply to economies of any size, due to the availability of public monitoring of defections.

\subsection{Control condition}

Define a generic meeting in round $t$ by $\left\{i, o_{i}(t)\right\}$, where $i$ is a player and $o_{i}(t)$ is the other player in the pair. To support full cooperation as an equilibrium outcome we consider a trigger strategy described by an automaton with two states, I and II.

Definition 1 (Cooperative strategy). At the start of any round $t$, player $i$ can be in state I or II, and takes actions only as a producer. As a producer, player $i$ selects "give help" in state $I$, and "no help" in state II. In $t=1$, the state is $I$; in all $t \geq 1$

(i) if player $i$ is in state $I$, then $i$ moves to state II in $t+1$ only if some producer in the group-not necessarily the producer in $\left\{i, o_{i}(t)\right\}$-chooses "no help." Otherwise, player $i$ remains in state I;

(ii) there is no exit from state II.

If this strategy is commonly adopted, then it is called a social norm. This social norm can support full cooperation in groups of any size. Intuitively, this norm consists of a rule of cooperation and rule for punishment: (i) Cooperation: if the player is a producer, then he selects "give help"; (ii) Punishment: if a defection is observed in the group, then the player will always select "no help" whenever he is a producer. The central feature of this norm is that the entire 
group participates in enforcing defections. In equilibrium no one defects. In what follows we show that, under this social norm, cooperation is a sequential equilibrium if the players' discount factor $\beta$ is sufficiently large.

Proposition 1. If $\beta \geq \beta^{*}:=\frac{d}{k-d+l}$, then the strategy in Definition 1 supports full cooperation in sequential equilibrium.

The proof is constructed by means of two lemmas. We start by calculating equilibrium payoffs. Recall that players deterministically alternate between the two roles of producer and consumer. Hence, in equilibrium players earn $k$ every other round. Discounting starts on date $T$, when the random termination rule starts; hence, only payoffs from rounds $t=T+1$ (included) are discounted at rate $\beta$. Let $v_{s}(t)$ denote the equilibrium payoff at the start of $t=1,2, \ldots$ to a player who is in role $s=0,1$, where $0=$ producer and $1=$ consumer.

Lemma 1. Fix $T \geq 1$ and $\beta \in(0,1)$. In the cooperative equilibrium we have $v_{1}(t)>v_{0}(t)$ for all $t=1,2, \ldots$, where for $h=1,2 \ldots$,

$$
v_{s}(t):= \begin{cases}k \times \frac{T-t}{2}+v_{s}, & \text { if } T-t=2 h \\ k \times \frac{T-t+1}{2}+\beta v_{s}, & \text { if } T-t=2 h-1, \\ v_{s}, & \text { if } T-t \leq 0,\end{cases}
$$

and

$$
v_{s}:=\frac{\beta^{1-s}}{1-\beta^{2}} \times k \quad \text { for } s=0,1 .
$$

Proof. See Appendix.

The equilibrium payoff is found by substituting $t=1$ in expression (1). To determine the optimality of the cooperative strategy we must check two items: (i) in equilibrium no producer has an incentive to defect; (ii) out of 
equilibrium no producer has an incentive to cooperate. We let $\hat{v}_{s}(t)$ denote the continuation payoff to a player in role $s$ on date $t$, off equilibrium.

Consider a generic producer in a round $t \geq 1$. In equilibrium, choosing "give help" is a best response if

$$
v_{0}(t) \geq \hat{v}_{0}(t)
$$

The left-hand-side of the inequality denotes the payoff to a producer who cooperates in the round, choosing "give help." The right-hand-side denotes the continuation payoff on date $t$ if the producer defects in equilibrium (reverting back to playing the social norm in the following round), given that off-equilibrium everyone follows the group punishment rule prescribed by the social norm. Hence, if a defection occurs on $t$, then every producer selects "no help" from $t+1$ because equilibrium defections are public.

It should be clear that

$$
\hat{v}_{0}(t)=\hat{v}_{0}:=\frac{d+\beta(d-l)}{1-\beta^{2}} \quad \text { if } t \geq T .
$$

For $h=1,2, \ldots$, the continuation payoff off-equilibrium satisfies

$$
\hat{v}_{0}(t):= \begin{cases}(d+d-l) \times \frac{T-t}{2}+\hat{v}_{0} & \text { if } T-t=2 h \\ (d+d-l) \times \frac{T-t+1}{2}+\beta \hat{v}_{0} & \text { if } T-t=2 h-1, \\ \hat{v}_{0} & \text { if } T-t \leq 0 .\end{cases}
$$

Off equilibrium payoffs are independent of the size of the group $N$ since producers defect forever after seeing a defection.

Lemma 2. Fix $T \geq 1$ and $\beta \in(0,1)$. If $\beta \geq \beta^{*}:=\frac{d}{k-d+l}$, then $v_{0}(t) \geq$ $\hat{v}_{0}(t)$ for all $t \geq 1$. 
Proof. See Appendix.

Given that everyone else follows the strategy in Definition 1, it is always individually optimal to punish out of equilibrium, because "no help" is the dominant action when everyone forever defects.

Note that $\hat{v}_{s}(1)$ is the payoff associated to infinite repetition of the static Nash equilibrium (every producer chooses "no help"), which is always an equilibrium of the repeated game. The condition $\beta \geq \beta^{*}$ is therefore necessary and sufficient for existence of a cooperative equilibrium because it ensures that players earn payoffs above those guaranteed by defecting in any round. The condition $\beta \geq \beta^{*}$ does not guarantee that cooperation will be realized because many equilibria exist in the game. Given the experimental parameters, $\beta^{*}=0.4$ in large groups and $\beta^{*}=0.5$ in partnerships. Hence, if participants are risk-neutral, then the fully cooperative equilibrium exists in the CONTROL condition, in groups of any size, because in the experiment $\beta=0.75$.

\subsection{Tokens condition}

All the equilibria that exist in the CONTROL condition also exists in the TOKENS condition, because tokens are intrinsically worthless, do not restrict action sets, and can be ignored. In addition, cooperation can be supported as an equilibrium by means of monetary trade.

Definition 2 (Monetary trade strategy). In any round $t$, after any history, if the player has no tokens, she has no action to take as a consumer and chooses "sell help" as a producer. If the player has some tokens, she chooses "buy help" as a consumer and selects "no help" as a producer.

We call monetary trade the outcome that results when everyone adopts the strategy in Definition 2. Here, help is only given quid-pro-quo in exchange for 
a token. Otherwise, help is not given. Monetary trade sustains the socially efficient allocation on the same parameter set as the social norm. The reason is that in monetary equilibrium all encounters support trade due to the deterministic alternation between roles.

Proposition 2. If $\beta \geq \beta^{*}$, then the monetary trade strategy in Definition 2 supports full cooperation in equilibrium.

Proof. See Appendix.

\section{Experimental procedures}

Sessions consisted of six cycles that lasted an average of 18.7 rounds of play. Cycle duration varied across cycles and sessions, but was identical for all groups in the same session. The size of groups in the four cycles of the Training Phase followed either the order 2-12-2-12 or 12-2-12-2. During the Selection Phase (cycles 5-6) the group size was determined endogenously by majority rule as follows. Before cycles 2-5, participants expressed a preference for groups of size 2 or 12 . These preferences were all counted in order to select the group size for cycle 5 . Before cycle 6, participants expressed a preference for groups of size 2 or 24 in cycle 6 .

The experiment involved 384 undergraduate volunteers, each of whom participated in only one session. Between September and October 2014, we ran 8 sessions for the Control and 8 for the Tokens condition, with 24 participants each. The conversion rate was 1 CUs $=U S \$ 0.20$. Sessions lasted 2.5 hours on average, and participants were paid on average US $\$ 26.73$ in cash, privately, at the end of the session. Only one randomly selected cycle from the session was paid. 
In each round, participants observed own payoff, tokens held (Tokens conditions), and if all producers in their group helped or not. Participants had continual access to such feedback from all past rounds of the cycle. They were informationally isolated across groups and no one interacted with any person met in previous cycles (except possibly in cycle 6). The experiment was programmed using the software z-Tree (Fischbacher, 2007) and ran at the laboratory in the Economic Science Institute at Chapman University. No eye contact was possible. Participants' demographic characteristics were collected through an end-of-session anonymous survey.

The experimenter read the instructions and participants followed on individual copies. The instructions adopted a neutral language: the words "help," "cooperation," and "money" were never used (see Appendix B). Before the Training Phase, participants took a quiz with ten questions testing their understanding of the instructions, and received 25 cents for each correct answer.

\section{Results}

Before presenting the two main results, based on the behavior in the Selection Phase, we provide an overview of behavior in the Training Phase.

In the Training Phase, average cooperation rates were higher in partnerships than in large groups $(69.4 \%$ vs. $50.0 \%$ in ConTroL; $67.6 \%$ vs. $48.8 \%$ in Tokens; p-value $<0.001$; Table A2 in Appendix A). However, partnerships did not create more surplus than large groups because of their lower potential (efficiency was $46.2 \%$ vs. $50.0 \%$ in CONTROL, and $46.1 \%$ vs. $48.8 \%$ in Tokens; p-value > 0.1; Table A2 in Appendix A).

While efficiency levels were similar across conditions, observed actions differed. Whenever monetary trade was possible in Tokens, consumers over- 
whelmingly chose "buy help" (81.8\%) and producers mostly chose "sell help" (63.4\%). Help was rarely given when consumers had no token (18.3\%); this contrasts with behavior observed under the same decisional situation in CoNTROL, where "give help" was the predominant choice (59.7\%). These differences across conditions during the Training Phase influenced the participants' willingness to widen the scale of interaction in the Selection Phase.

Result 1. Money promotes the formation of large groups.

Participants in ToKens selected to interact in large groups more frequently than in Control (Table A3 in Appendix A). In the Selection Phase the difference is statistically significant (55.8\% vs. $39.3 \%$, p-value 0.014 ; Table A4 in Appendix A).

Two elements of the experience during the Training Phase determined the participants' disposition to widen the scale of interaction: experiences of exploitation by free-riders and of full cooperation. We measure exploitation as the help imbalance, which is the difference between how frequently a participant gave and received help in a cycle (Figure 1). The imbalance goes from -1 to 1 : it is negative for someone who gave help more frequently than she received it, positive otherwise. The imbalance is zero for someone who equally gave and received help, which occurs with full cooperation (dark bars in Figure 1), no cooperation, and some instances of partial cooperation.

Participants are unsure which strategy others will use. This strategic uncertainty (Van Huyk et al., 2007) implies that those who help in order to establish a cooperative norm may not receive help in future rounds. This exploitation hazard is captured by the dispersion of help imbalance across participants; Figure 1 reveals that it was greater in large groups than partnerships. A zero imbalance was more frequently attained in partnerships than large groups 
(0.563 vs. 0.156 in Control, p-value $<0.001,0.609$ vs. 0.299 in Tokens, p-value < 0.001; Table A5 in Appendix A), and also in Tokens than ConTROL, especially in large groups where it was almost twice as frequent (0.299 vs. 0.156, p-value < 0.001; Table A6 in Appendix A). The possibility to trade tokens for help quid-pro-quo offers protection against exploitation hazards: a participant must transfer a token to receive help, and the only way to obtain tokens is to help others.
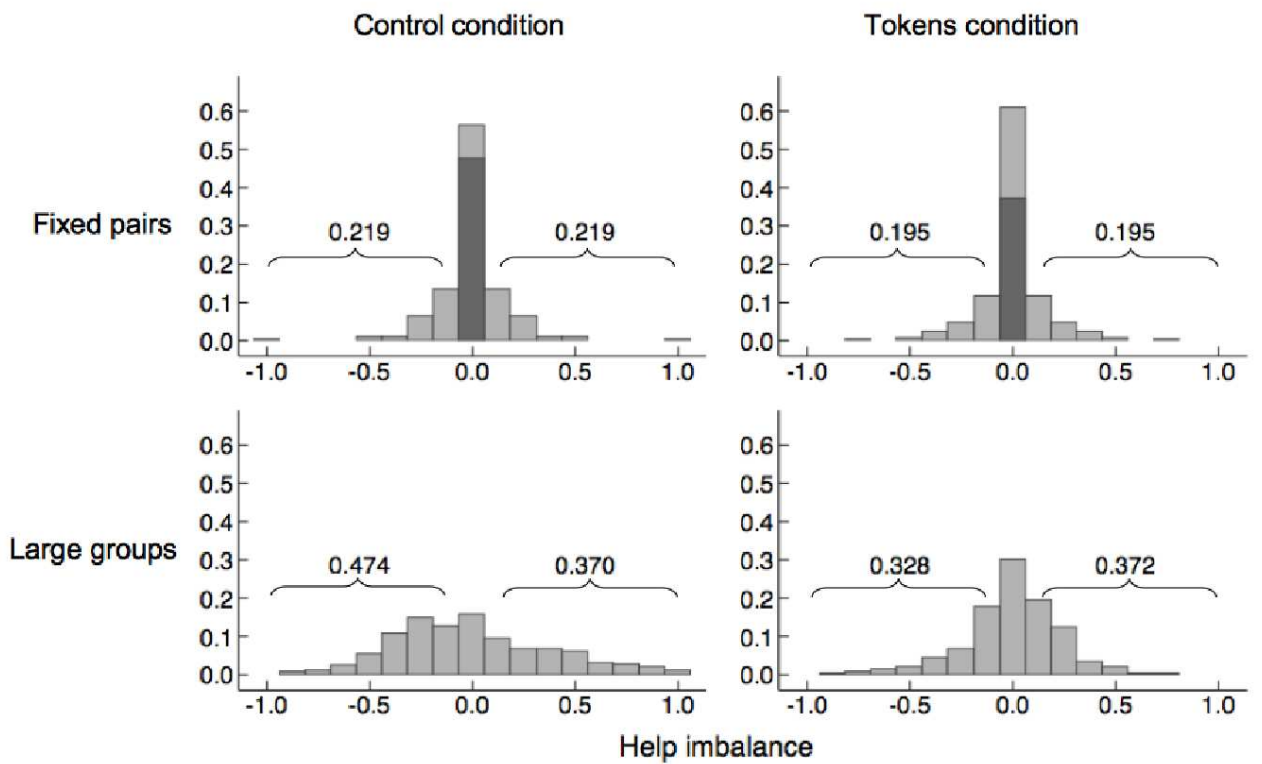

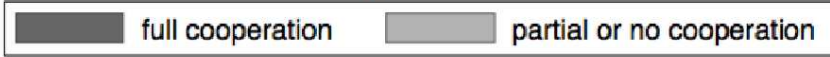

Figure 1: The distribution of help imbalance.

Notes: Help imbalance is the difference between how frequently a participant gave and received help in a cycle. Participants who gave more (less) help than they received have a negative (positive) imbalance. Data from rounds 1-16, Training Phase only. Four observations per participant.

The probit regression in Table 1 estimates how the desire to widen the scale of interaction is affected by help imbalance and full cooperation experienced in 
partnerships and groups of strangers. The dependent variable takes value 1 when a participant expressed a preference for large groups of 12 and 24 (cycles 5 and 6 , respectively) and zero otherwise. This regression reveals that help imbalance in large groups is crucial. Those who received more help than they gave, i.e. the free riders, were more willing to interact in large groups, where they could not be directly targeted for punishment. Instead, those exploited by free riders were more likely to opt for the safety experienced in partnerships.

The share of free riders was similar across conditions (37.0\% vs. $37.2 \%$, Figure 1), but more participants were exploited in Control than in Tokens (47.4\% vs. $32.8 \%$, Figure 1). This suggests that the differential experience of exploitation was behind the weaker desire to expand the scale of interaction in Control.

Table 1: Money reduces strategic uncertainty and promotes the formation of large groups.

\begin{tabular}{lcc}
\hline \hline Dependent variable: Individual preference & & \\
for large groups (0=partnerships) & marg. eff. & S.E \\
\hline Tokens condition x cycle 5 (dummy) & 0.112 & $(0.075)$ \\
Tokens condition x cycle 6 (dummy) & $0.153^{*}$ & $(0.079)$ \\
Cycle 6 (dummy) & -0.086 & $(0.053)$ \\
Training phase & & \\
Help imbalance - partnerships & 0.155 & $(0.149)$ \\
Help imbalance - large groups & $0.304^{* * *}$ & $(0.072)$ \\
Full cooperation - partnerships (dummy) & $-0.183^{* * *}$ & $(0.062)$ \\
Controls & Yes & \\
\hline N & 768 & \\
\hline
\end{tabular}

Notes: Probit regressions on preferences for large groups of 12 and 24 (cycles 5 and 6 , respectively). The regression includes controls for order effects in the Training Phase, sex, the number of right answers and response time in a comprehension test on the instructions. Marginal effects are computed at the regressors' mean value (at zero for dummy variables). One observation per person per cycle. Data from rounds 1-16 only.

Large groups never attained full cooperation, while several partnerships 
attained it (37.0\% in Tokens and $47.4 \%$ in Control, Figure 1). Those who were in a cooperative partnership were less willing to widen the scale of interaction than those in other partnerships (the regressor "Full cooperation" in Table 1 is negative and highly significant). Partners attained full cooperation more frequently in Control than in Tokens ( $\mathrm{p}$-value $=0.08$; Table A7 in Appendix A), which suggests that the possibility of relying on monetary trade displaced norms of voluntary help (Camera et al., 2013). This is a second reason behind the weaker desire to expand the scale of interaction observed in Control compared to Tokens.

The "Tokens condition" dummies capture the residual difference across conditions in participants' willingness to widen the scale of interaction. The estimated coefficient is positive and significant only for cycle 6 , when groups of 24 could be formed, but not for cycle 5, where the size of large groups was 12 , as in the Training Phase. A reason may be that participants never experienced interaction in groups of 24 before. In this case the presence of tokens made a difference, because participants realized that monetary trade reduced strategic uncertainty. That is why participants in TOKENS condition were more willing to select large groups.

Recall that, by design, cooperative large groups create $50 \%$ more surplus than cooperative partnerships, thus raising efficiency from $67 \%$ to $100 \%$. But uncooperative large groups may also destroy surplus relative to partnerships. Maximum efficiency could be attained in any condition by simply taking turns at helping others - it did not require the exchange of tokens. By contrast, experimental data reveal different patterns across conditions.

Result 2. Without tokens, endogenously-formed groups achieved lower efficiency than partnerships. The converse held true with tokens. 
In the experiment, wide disparities emerged between TOKEns and ConTROL in the Selection Phase - when the group size was endogenous. In CoNTROL, efficiency fell when participants chose to widen the scale of interaction. In Tokens, the opposite held true.

Table 2: How monetary trade and group size influence efficiency.

\begin{tabular}{llc}
\hline \hline Dependent variable: efficiency & coefficient & S.E \\
\hline Control $\times$ large & $-0.121^{*}$ & $(0.064)$ \\
Tokens $\times$ partnership & -0.021 & $(0.049)$ \\
Tokens $\times$ large & $0.101^{* *}$ & $(0.042)$ \\
Cycle 6 (dummy) & 0.014 & $(0.036)$ \\
Constant & $0.566^{* * *}$ & $(0.034)$ \\
\hline N & 32 & \\
R-squared & 0.343 & \\
\hline
\end{tabular}

Notes: One observation per session per cycles 5 and 6 . The default condition is Control, partnerships. Linear regression on realized efficiency on a set of dummy variables that include the interaction between condition and group size. Data from rounds 1-16, Selection Phase only.

The linear regression in Table 2 measures how efficiency varies with group size and availability of tokens. The dependent variable is the realized efficiency in a cycle, in a session. In Tokens large groups attained significantly greater efficiency than partnerships (67.2\% vs. 55.4\%, two-sided Wald test on the estimated coefficients, $\mathrm{p}$-value $=0.027)$. The opposite was true in ConTROL (45.0\% vs. $57.3 \%$ ). Large groups also attained greater efficiency in Tokens than Control (two-sided Wald test on the estimated coefficients, $\mathrm{p}$-value $=0.002)$. In partnerships, instead, efficiency levels were similar across conditions.

The distribution of efficiency across large groups gives us an additional measure of how monetary trade affected economic performance. In the ToKENS condition, 16 large groups were formed in the Selection Phase; half of these groups exceeded the $67 \%$ efficiency threshold of partnerships (Figure 2). 
Instead, in the ConTrol condition this happens only in 1 of the 5 large groups that were formed. Tokens are intrinsically worthless, so their availability did not raise efficiency per se. Tokens merely offered participants an additional way to support cooperation among strangers. In fact, efficiency systematically improved with the intensity of monetary trade (Figure 2). Those groups that established a solid convention of trade attained efficiency above partnerships, while those where the convention of monetary trade failed to take hold, attained efficiency below that of the average partnership. This positive relation holds for the Training and Selection Phases.

Linear regressions on average payoff per-round attained by participants in large groups (Selection Phase) show a positive and significant effect of the intensity of monetary trade at the group and at the individual level (Table 3). The dependent variable is the average payoff per-round for a participant in a large group ( 0,1 , or 2 observations per participant). The regressors include two variables related to the intensity of monetary trade: at the group and individual level. ${ }^{1}$

\section{Conclusions}

Economies prosper when their members move beyond local exchange and cooperate with outsiders in the creation of wealth. But widening the scale of cooperation presents formidable challenges: interaction becomes impersonal and reciprocity unfeasible, as trust and social norms are weakened. This paper studies a setup where money is theoretically inessential, and shows that

${ }^{1}$ The intensity of monetary trade at the group level is measured as the overall frequency of the actions "sell help" and "buy help"; at the individual level it is measured as the frequency of the actions "sell help" and "buy help" in all rounds in which monetary trade was possible (i.e. the consumer had at least one token). 


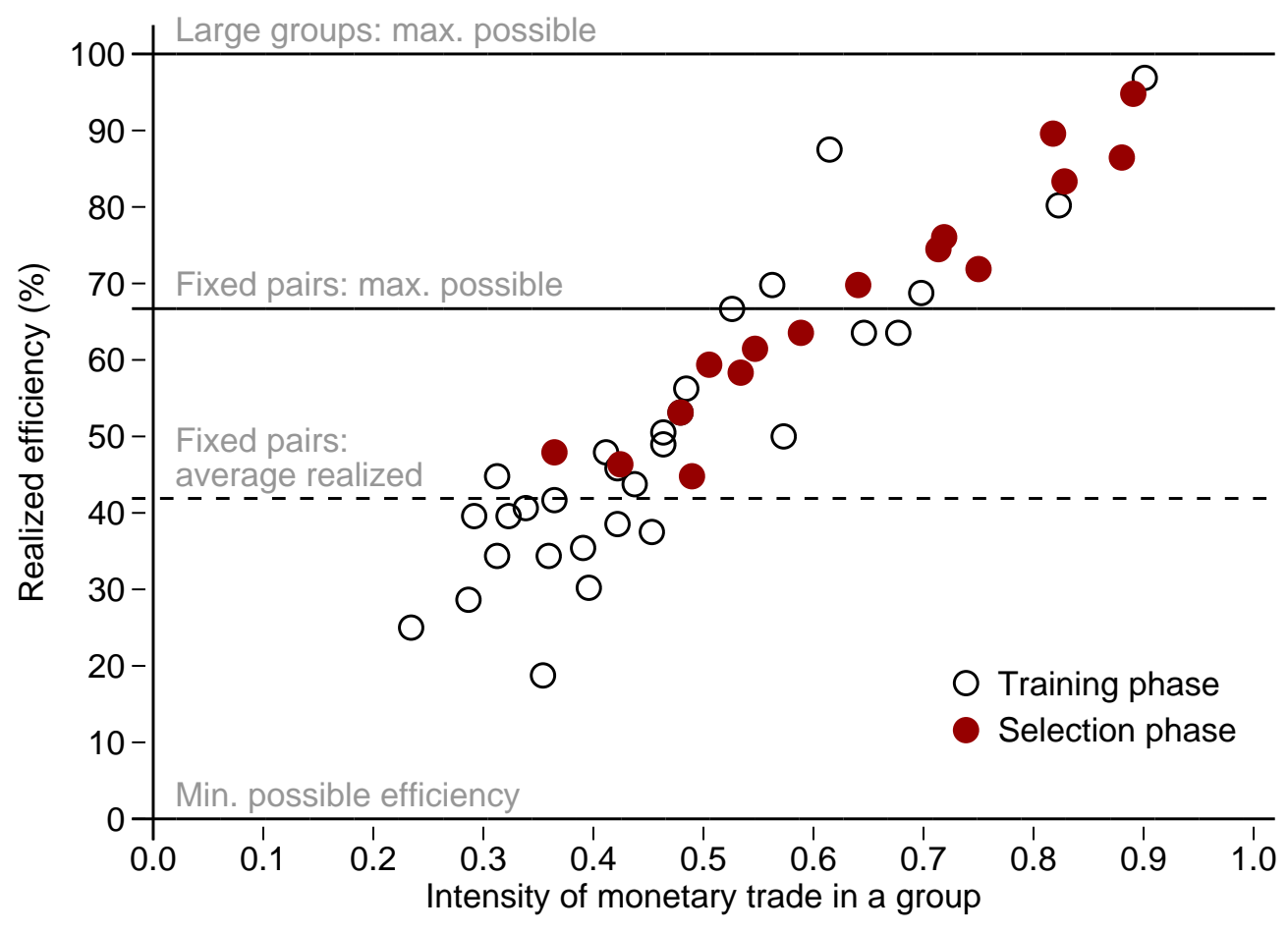

Figure 2: A strong monetary trade convention boosts efficiency in large groups.

Notes: One observation per group, per cycle. The intensity of monetary trade is the overall frequency of the actions "sell help" and "buy help." Minimum efficiency $(0 \%)$ is obtained when help is never given. Maximum efficiency in fixed-pairs is 67\%, which is obtained when help is always given; in large groups it is $100 \%$. Realized efficiency in partnerships (41.7\%) is computed aggregating data from the Training and Selection Phases (dashed line). Data from rounds 1-16, Tokens condition only. 
Table 3: Intense monetary trade raises payoffs in large groups.

\begin{tabular}{lcc}
\hline \hline $\begin{array}{l}\text { Dependent variable: } \\
\text { average per round profit }\end{array}$ & coefficient & S.E \\
\hline $\begin{array}{l}\text { Intensity of monetary trade } \\
\text { at the group level }\end{array}$ & $3.421^{* * *}$ & $(0.189)$ \\
$\quad \begin{array}{l}\text { at the individual level } \\
\text { Cycle 6 (dummy) }\end{array}$ & $-0.916^{* * *}$ & $(0.207)$ \\
Controls & & $(0.045)$ \\
Constant & $3.782^{* * *}$ & $(0.507)$ \\
\hline N & 240 & \\
R-squared & 0.413 & \\
\hline
\end{tabular}

Notes: Linear regression on data for large groups in the Selection Phase, Tokens condition. The dependent variable is the average payoff per-round for a participant in a large group. Among the regressors we include a dummy taking value one for cycle 6. The regression includes controls for order effects in the Training Phase, sex, the number of right answers and response time in a comprehension test on the instructions. Standard errors are robust for clustering at the session level. Data from rounds 1-16 only.

stable monetary systems are behaviorally crucial to expand the scale of cooperation.

In the experiment, participants faced an intertemporal cooperative task and could restrict interaction to partnerships, or expand it to large groups of strangers where the returns from cooperation were higher. When participants could trade symbolic tokens for cooperation, large groups spontaneously emerged, and created more surplus than partnerships. Instead, large groups rarely emerged without money and, when they did, free-riding prevailed.

Two remarks are in order. First, the exchange of symbolic objects is not necessary to sustain cooperation, in groups of any size. Second, the mere presence of tokens in the economy does not mechanically guarantee a cooperative outcome; participants in large groups must also be able to establish a strong convention of monetary trade.

This study also uncovers a key behavioral advantage of monetary exchange 
in promoting large-scale cooperation: it offers protection from strategic uncertainty. Participants are unsure about what others will choose because of equilibrium multiplicity ranging from zero to full cooperation. This uncertainty is the central stumbling block to widening the scale of cooperation. Participants realize that opportunistic temptations are stronger in large groups, because free-riders cannot be directly targeted for punishment, which raises uncertainty. In contrast, partners can rely on reciprocity and reputation and can also more easily coordinate on a common strategy compared to strangers in a large group. Choosing the scale of interaction thus hinges on the perceived trade-off between a partnership's low but predictable payoff, and the possibly higher but unpredictable payoff of large groups.

A monetary trade convention reduces strategic uncertainty because it prevents free-riders from exploiting cooperators: producers help only in exchange for a token, and only consumers who helped in the past have a token. Hence, the monetary strategy supports full cooperation through a unique rule of behavior on- and off-equilibrium. In contrast, a social norm of mutual help has two separate components: cooperation and punishment, and a coordination challenge arises from the existence of multiple ways to punish.

This insight open new avenues for theoretical advances. A monetary trade convention empirically outperforms a social norm of mutual help because it facilitates off-equilibrium coordination. However, the problem of coordination out-of-equilibrium is typically neglected in the theory. This consideration could lead to new theoretical models with sharper empirical predictions. 


\section{References}

T.K. Ahn, R. M. Isaac, and T. C. Salmon, 2009. Coming and going: Experiments on endogenous group sizes for excludable public goods. J. Pub. Econ. 93(1-2), 336-351.

M. Bigoni, G. Camera, and M. Casari, 2013. Money is More than Memory. Working Paper No. 14-17, Economic Science Institute, Chapman University.

G. Camera, and M. Casari, 2014. The Coordination Value of Monetary Exchange: Experimental Evidence. Am. Econ. J.: Micro., 6(1), 290-314.

G. Camera, M. Casari, and M. Bigoni, 2013. Money and trust among strangers. Proc. Natl. Acad. Sci. U.S.A., 110(37), 14889-14893.

P. Dal Bó, and G. Fréchette, 2011. The Evolution of Cooperation in Infinitely Repeated Games: Experimental Evidence. Am. Econ. Rev., 101(1), 411-429.

J. Duffy and D. Puzzello, 2014. Gift Exchange versus Monetary Exchange: Theory and Evidence. Am. Econ. Rev., 104(6), 1735-1776.

G. Ellison, 1994. Cooperation in the Prisoner's Dilemma with Anonymous Random Matching, Review of Economic Studies, 61(3), 567-588.

E. Fehr and S. Gächter, 2000. Fairness and retaliation: the economics of reciprocity, Journal of Economic Perspective, 14(3), 159-181.

U. Fischbacher, 2007. Z-Tree: Zurich toolbox for ready-made economic experiments, Experimental economics, 10(2), 171-178.

G. Fréchette and S. Yuksel, 2014. Infinitely Repeated Games in the Laboratory: Four Perspectives on Discounting and Random Termination. Working paper, New York University.

J. W. Friedman, 1971. A non-cooperative equilibrium for supergames. Rev. Econ. Stud., 1-12.

S. Gächter and B. Herrmann. 2011. The limits of self-governance when cooperators get punished: Experimental evidence from urban and rural Russia. European Economic Review 55(2), 193-210.

A. Greif, 2006. The Birth of Impersonal Exchange: The Community Responsibility System and Impartial Justice. J. Econ. Persp., 20(2), 221-236. 
M. Kandori, 1992. Social Norms and Community Enforcement, Review of Economic Studies, vol. 59(1), 63-80.

E. O. Kimbrough, V. Smith and B. J. Wilson, 2008. Historical Property Rights, Sociality, and the Emergence of Impersonal Exchange in Long-Distance Trade. Am. Econ. Rev., 98(3), 1009-1039.

P. Lian, and C. R. Plott, (1998) General equilibrium, markets, macroeconomics, and money in a laboratory experimental environment. Econ. Th., $12(1), 21-75$.

R. S. Lopez, 1971. The Commercial Revolution of the Middle Ages, 950-1350. Englewood Cliffs, N.J., Prentice-Hall.

K. A. McCabe, 1989. Fiat money as a store of value in an experimental market. Journal of Economic Behavior \& Organization, 12(2), 215-231.

P. R. Milgrom, D. C. North, and B. R. Weingast, 1990. The Role of Institutions in the Revival of Trade: The Law Merchant, Private Judges, and the Champagne Fairs. Econ. and Pol., 2(1), 1-23.

D.C. North, 1991. Institutions. J. Econ. Persp., 5(1), 97-112.

T. R. Palfrey, and H. Rosenthal, 1994. Repeated Play, Cooperation and Coordination: An Experimental Study. Rev. Econ. Stud., 61(3), 545-565.

C. Plott, 2001. Equilibrium, Equilibration, Information and Multiple Markets: From Basic Science to Institutional Design. Nobel Symposium on Behavioral and Experimental Economics, Stockholm, Sweden.

A. E. Roth, and K. Murnighan. 1978. Equilibrium behavior and repeated play of the prisoner's dilemma. J. Math. Psy., 17, 189-98

R. Townsend, 1980. Models of money with spatially separated agents. In: Kareken, J., Wallace, N. (eds.) Models of monetary economies, 265-303. Minneapolis: Federal Reserve Bank of Minneapolis.

J. Van Huyk, R. Battalio, and R. Beil, 1990. Tacit Coordination Games, Strategic Uncertainty, and Coordination Failure. Am. Econ. Rev. 80, 234248.

R. A. Weber, 2006. Managing Growth to Achieve Efficient Coordination in Large Groups. Am. Econ. Rev., 96(1), 114-126. 


\section{Appendix}

Proof of Lemma 1. To prove the result we consider the two cases $t \geq T$ and $t<T$ separately.

Let $v_{s}$ denote the equilibrium payoff at the start of round $t \geq T$ to a player who is in role $s=0,1$ ( 0 identifies a producer). It holds that

$$
v_{s}:=\frac{\beta^{1-s}}{1-\beta^{2}} \times k \quad \text { for } s=0,1
$$

The payoff is time invariant due to the stationary alternation between roles.

Now consider round $t<T$. Given the proposed strategy those who are initial consumers earn $k$ on odd dates $(t=1,3, \ldots)$ and zero otherwise; initial producers earn $k$ on even dates $(t=2,4, \ldots)$ and zero otherwise. Hence, knowing whether $T-t$ is odd or even matters. For $j, h=1,2 \ldots$ and $s=0,1$ it holds that

$$
v_{s}(t)= \begin{cases}k \times \frac{T-t}{2}+v_{s} & \text { if } T-t=2 h \\ k \times \frac{T-t+1}{2}+\beta v_{s} & \text { if } T-t=2 h-1 .\end{cases}
$$

The continuation payoff $v_{s}(t)$ has two components. The first sums up the round payoffs for all $t \leq T-1$. The second sums up the round payoffs for all $t \geq T$. It should be clear that $v_{s}(t)$ is increasing in $T$ for $s=0,1$ and it achieves a minimum when $T-t=1$. Hence, the equilibrium payoff to a player in role $s=0,1$ on any date $t \geq 1$ is given by (1). We have $v_{1}(t)>v_{0}(t)$ for all $t$ because $v_{1}>v_{0}$ for all $\beta \in(0,1)$.

Proof of Lemma 2. The result is obtained by manipulation of the equations 
in (3). Note that

$$
v_{0}-\hat{v}_{0}=\frac{\beta}{1-\beta^{2}} \times k-\frac{d+\beta(d-l)}{1-\beta^{2}}=\frac{\beta}{1-\beta^{2}} \times(k-2 d+l)-\frac{d}{1+\beta}
$$

Now define

$$
\begin{aligned}
\Delta_{0}(t) & =v_{0}(t)-\hat{v}_{0}(t) \\
& = \begin{cases}(k-2 d+l) \times \frac{T-t}{2}+v_{0}-\hat{v}_{0} & \text { if } T-t=2 h \\
(k-2 d+l) \times \frac{T-t+1}{2}+\beta\left(v_{0}-\hat{v}_{0}\right) & \text { if } T-t=2 h-1, \\
v_{0}-\hat{v}_{0} & \text { if } T-t \leq 0 .\end{cases}
\end{aligned}
$$

It is immediate that $\Delta_{0}(t=T-2 h)>\Delta_{0}(t \geq T)$; note that $k-2 d+l>0$ by assumption. Also, $\Delta_{0}(t=T-2 h+1)>\Delta_{0}(t \geq T)$; to prove it insert $h=1$ (the most stringent case), rearrange the inequality, and then insert the expression for $v_{0}-\hat{v}_{0}$, to obtain the inequality $k-2 d+l>-d$.

Given that the minimum value of $\Delta_{0}(t)$ is achieved for $T-t \leq 0$, then (2) holds for all $t$ whenever

$$
\begin{aligned}
0 & \leq v_{0}-\hat{v}_{0}=\frac{\beta}{1-\beta^{2}} \times(k-2 d+l)-\frac{d}{1+\beta} \\
& \Leftrightarrow \beta \geq \beta^{*}:=\frac{d}{k-d+l} .
\end{aligned}
$$

Note that $\beta^{*}<1$ because $k-2 d+l>0$ by assumption.

Proof of Proposition 2. Conjecture that monetary trade is an equilibrium. Consider a player with $s=0,1$ tokens at the start of a round. In equilibrium, a consumer has a token and a producer has none. Hence, the probability that a consumer with a token meets a producer without tokens is 1 . Denote by $v_{s}(t)$ the equilibrium continuation payoff. Because the consumption pattern is 
the same as under the social norm, in monetary equilibrium it holds that $v_{s}(t)$ corresponds to the functions defined in (1).

Now consider deviations. We start by proving that a consumer does not deviate in equilibrium, refusing quid-pro-quo exchange for help. In round $t \geq 1$ let $\beta_{t}=1$ if $t<T$ and $\beta_{t}=\beta$ otherwise. Denote by $\tilde{v}_{1}(t)$ the payoff in $t$ to a consumer who defects by refusing to spend money in $t$. Using recursive arguments we have

$$
\begin{aligned}
\tilde{v}_{1}(t) & =d-l+\beta_{t}\left[d+\beta_{t+1} v_{1}(t+2)\right] \\
& <k+\beta_{t}\left[0+\beta_{t+1} v_{1}(t+2)\right]=v_{1}(t)
\end{aligned}
$$

The inequality holds for any $\beta_{t}$ because $k>d+d-l$ by assumption. To understand the inequality consider the first line. Defecting in $t$ generates payoff $d-l$ instead of $k$, and in $t+1$ the player will be a producer with money, reverting back to playing the monetary strategy (unimprovability criterion). Hence, she will refuse to sell for another token because she already has one; this is optimal because (i) acquiring an additional token costs her $d$ and (ii) she has already one token to spend. hence, in $t+2$ the player becomes a consumer with money and the distribution of tokens is back at equilibrium. In summary, following a unilateral deviation in $t$ by a consumer, the group is back on the equilibrium path in round $t+2$.

Now we prove that if $\beta \geq \beta^{*}$, then a producer in equilibrium would not want to deviate in any $t$, refusing to help for a token. Denote by $\tilde{v}_{0}(t)$ the payoff in $t$ to a producer who defects by refusing to accept money in $t$. Using 
recursive arguments, we have

$$
\begin{aligned}
\tilde{v}_{0}(t) & =d+\beta_{t}\left[d-l+\beta_{t+1} v_{0}(t+2)\right] \\
& <0+\beta_{t}\left[k+\beta_{t+1} v_{0}(t+2)\right]=v_{0}(t)
\end{aligned}
$$

The inequality holds for any $\beta_{t} \geq \beta^{*}$ because $k>d+d-l$ (if $\beta_{t}=1$ ); if $\beta_{t}=\beta$, then we need $\beta \geq \beta^{*}$. The first line of the inequality shows that defecting in $t$ generates payoff $d$ instead of 0 . In $t+1$ the player is a consumer without money; she cannot buy help - since everyone follows the monetary strategyand earns $d-l$. In $t+2$ she is a producer without money and the distribution of tokens is back at equilibrium. Hence, after a unilateral deviation in $t$ by a producer, the group is back in equilibrium in round $t+2$. 